Upgrade of the CMS muon system with triple-GEM detectors

This content has been downloaded from IOPscience. Please scroll down to see the full text. 2014 JINST 9 C10036

(http://iopscience.iop.org/1748-0221/9/10/C10036)

View the table of contents for this issue, or go to the journal homepage for more

Download details:

IP Address: 157.193.98.234

This content was downloaded on 11/06/2015 at 14:02

Please note that terms and conditions apply. 


\section{Upgrade of the CMS muon system with triple-GEM detectors}

D. Abbaneo, ${ }^{o}$ M. Abbas, ${ }^{o}$ M. Abbrescia, ${ }^{b}$ A.A. Abdelalim, ${ }^{h}$ M. Abi Akl, ${ }^{m}$ W. Ahmed, ${ }^{h}$ W. Ahmed, ${ }^{q}$ P. Altieri, ${ }^{b}$ R. Aly, ${ }^{h}$ A. Ashfaq, ${ }^{q}$ P. Aspell, ${ }^{o}$ Y. Assran, ${ }^{g}$ I. Awan, ${ }^{q}$ S. Bally, ${ }^{o}$ Y. Ban, ${ }^{c}$ S. Banerjee, ${ }^{s}$ P. Barria, ${ }^{e}$ L. Benussi, ${ }^{n}$ V. Bhopatkar, ${ }^{v}$ S. Bianco, ${ }^{n}$ J. Bos, ${ }^{o}$ O. Bouhali, ${ }^{m}$ S. Braibant ${ }^{d}$ S. Buontempo, ${ }^{x}$ J. Cai, ${ }^{c}$ C. Calabria, ${ }^{b}$ C. Caputo, ${ }^{b}$

F. Cassese,${ }^{x}$ A. Castaneda, ${ }^{m}$ S. Cauwenbergh,${ }^{p}$ F.R. Cavallo, ${ }^{d}$ A. Celik, ${ }^{i}$ M. Choi, ${ }^{a e}$ K. Choi, ${ }^{a e}$ S. Choi, ${ }^{a c}$ J. Christiansen, ${ }^{o}$ A. Cimmino, ${ }^{p}$ S. Colafranceschi, ${ }^{o}$ A. Colaleo, ${ }^{b}$ A. Conde Garcia, ${ }^{o}$ M.M. Dabrowski, ${ }^{o}$ G. De Lentdecker, ${ }^{e}$ R. De Oliveira, ${ }^{o}$ G. de Robertis, ${ }^{b}$ S. Dildick, ${ }^{i, p}, 1$ B. Dorney, ${ }^{o}$ W. Elmetenawee, ${ }^{h}$ G. Fabrice, ${ }^{a}{ }^{a}$ S. Ferry, ${ }^{o}$ P. Giacomelli, ${ }^{d}$ J. Gilmore ${ }^{i}$ L. Guiducci, ${ }^{d}$ A. Gutierrez, ${ }^{l}$ R.M. Hadjiiska, ${ }^{a}{ }^{b}$ A. Hassan, ${ }^{h}$ J. Hauser ${ }^{u}$ K. Hoepfner, ${ }^{a}$ M. Hohlmann, ${ }^{\nu}$ H. Hoorani, ${ }^{q}$ Y.G. Jeng, ${ }^{r}$ T. Kamon, ${ }^{i}$ P.E. Karchin, ${ }^{l}$ H. Kim, ${ }^{r}$ S. Krutelyov, ${ }^{i}$ A. Kumar, ${ }^{k}$ J. Lee,,${ }^{a e}$ J. Lee, ${ }^{a e}$ T. Lenzi, ${ }^{e}$ L. Litov, ${ }^{a b}$ F. Loddo, ${ }^{b}$ T. Maerschalk, ${ }^{e}$ G. Magazzu, ${ }^{z}$ M. Maggi, ${ }^{b}$ Y. Maghrbi, ${ }^{m}$ A. Magnani, ${ }^{y}$ N. Majumdar, ${ }^{s}$ P.K. Mal, ${ }^{f}$ K. Mandal, ${ }^{f}$ A. Marchioro, ${ }^{o}$ A. Marinov, ${ }^{o}$ J.A. Merlin, ${ }^{o}$ N. Mohammed, ${ }^{k}$ A.K. Mohanty, ${ }^{w}$ A. Mohapatra, ${ }^{v}$ S. Muhammad, ${ }^{q}$ S. Mukhopadhyay, ${ }^{s}$ S. Nuzzo, ${ }^{b}$ E. Oliveri, ${ }^{o}$ L.M. Pant, ${ }^{w}$ P. Paolucci, ${ }^{x}$ I. Park, ${ }^{a}$ G. Passeggio, ${ }^{x}$ B. Pavlov, ${ }^{a b}$ B. Philipps, ${ }^{a}$ M. Phipps, ${ }^{,}$D. Piccolo, ${ }^{n}$ H. Postema, ${ }^{o}$ G. Pugliese,${ }^{b}$ A. Puig Baranac, ${ }^{o}$ A. Radi, ${ }^{g}$ R. Radogna,${ }^{b}$ G. Raffone,${ }^{n}$

S. Ramkrishna, ${ }^{k}$ A. Ranieri, ${ }^{b}$ C. Riccardi, ${ }^{y}$ A. Rodrigues, ${ }^{o}$ L. Ropelewski, ${ }^{o}$ S. Roychoddhury, ${ }^{s}$ M.S. Ryu, ${ }^{r}$ G. Ryu, ${ }^{a e}$ A. Safonov, ${ }^{i}$ A. Sakharov, ${ }^{j}$ S. Salva, ${ }^{p}$ G. Saviano, ${ }^{n}$ A. Sharma, ${ }^{o}$ S.K. Swain, ${ }^{f}$ J.P. Talvitie, ${ }^{o}$, C. Tamma,${ }^{b}$ A. Tatarinov, ${ }^{i}$ N. Turini, ${ }^{z}$ T. Tuuva, ${ }^{t}$ J. Twigger, ${ }^{v}$ M. Tytgat,${ }^{p}$ I. Vai, ${ }^{y}$ M. van Stenis,${ }^{o}$ R. Venditi, ${ }^{b}$ E. Verhagen, ${ }^{e}$ P. Verwilligen, ${ }^{b}$ P. Vitulo, ${ }^{y}$ U. Yang, ${ }^{a d}$ Y. Yang, ${ }^{e}$ R. Yonamine,${ }^{e}$ N. Zaganidis, ${ }^{p}$ F. Zenoni ${ }^{e}$ and A. Zhang ${ }^{v}$

${ }^{a}$ III Physikalisches Institut A, RWTH Aachen University, Aachen, Germany

${ }^{b}$ Politecnico di Bari, Universitá di Bari and INFN Sezione di Bari, Bari, Italy

\footnotetext{
${ }^{1}$ Corresponding author.
} 
${ }^{c}$ Peking University, Beijing, China

${ }^{d}$ University and INFN Bologna, Bologna, Italy

${ }^{e}$ Université Libre de Bruxelles, Brussels, Belgium

${ }^{f}$ National Institute of Science Education and Research, Bhubaneswar, India

${ }^{g}$ Academy of Scientific Research and Technology, ENHEP, Cairo, Egypt

${ }^{h}$ Helwan University \& CTP, Cairo, Egypt

${ }^{i}$ Texas A\&M University, College Station, U.S.A.

${ }^{j}$ Kyungpook National University, Daegu, Korea

${ }^{k}$ University of Delhi, Delhi, India

${ }^{l}$ Wayne State University, Detroit, U.S.A.

${ }^{m}$ Texas A\&M University at Qatar, Doha, Qatar

${ }^{n}$ Laboratori Nazionali di Frascati - INFN, Frascati, Italy

${ }^{\circ}$ CERN, Geneva, Switzerland

${ }^{p}$ Department of Physics and Astronomy, Ghent University, Ghent, Belgium

${ }^{q}$ National Center for Physics, Quaid-i-Azam University Campus, Islamabad, Pakistan

${ }^{r}$ Chonbuk National University, Jeonju, Korea

${ }^{s}$ Saha Institute of Nuclear Physics, Kolkata, India

${ }^{t}$ Lappeenranta University of Technology, Lappeenranta, Finland

"University of California, Los Angeles, U.S.A.

${ }^{v}$ Florida Institute of Technology, Melbourne, U.S.A.

${ }^{w}$ Bhabha Atomic Research Centre, Mumbai, India

${ }^{x}$ INFN Napoli, Napoli, Italy

${ }^{y}$ INFN Pavia and University of Pavia, Pavia, Italy

${ }^{z}$ INFN Sezione di Pisa, Pisa, Italy

aa IRFU CEA-Saclay, Saclay, France

${ }^{a b}$ Sofia University, Sofia, Bulgaria

${ }^{a c}$ Korea University, Seoul, Korea

${ }^{a d}$ Seoul National University, Seoul, Korea

${ }^{a e}$ University of Seoul, Seoul, Korea

E-mail: sven.dildick@ugent.be

ABSTRACT: The CMS collaboration considers upgrading the muon forward region which is particularly affected by the high-luminosity conditions at the LHC. The proposal involves Gas Electron Multiplier (GEM) chambers, which are able to handle the extreme particle rates expected in this region along with a high spatial resolution. This allows to combine tracking and triggering capabilities, which will improve the CMS muon High Level Trigger, the muon identification and the track reconstruction. Intense $R \& D$ has been going on since 2009 and it has lead to the development of several GEM prototypes and associated detector electronics. These GEM prototypes have been subjected to extensive tests in the laboratory and in test beams at the CERN Super Proton Synchrotron (SPS). This contribution will review the status of the CMS upgrade project with GEMs and its impact on the CMS performance.

KEYWORDS: Micropattern gaseous detectors (MSGC, GEM, THGEM, RETHGEM, MHSP, MICROPIC, MICROMEGAS, InGrid, etc); Electron multipliers (gas) 


\section{Contents}

1 Introduction 1

2 The CMS GEM project 2

3 Gaseous electron multipliers $\quad 2$

4 Impact on trigger and physics impact at the high-luminosity LHC 3

4.1 Trigger 3

4.2 Physics 4

5 R\&D for CMS GEM project $\quad 4$

5.1 Early GEM prototypes 4

5.2 Large GEM prototypes 4

5.3 GEM foil stretching 5

5.4 Aging tests 5

$\begin{array}{ll}\text { 5.5 Chamber construction and quality control } & 6\end{array}$

$\begin{array}{ll}\text { 5.6 Data acquisition and electronics } & 6\end{array}$

$\begin{array}{lll}6 & \text { Integration into CMS } & 7\end{array}$

7 Conclusions and outlook $\quad 7$

\section{Introduction}

The Compact Muon Solenoid [1] at the Large Hadron Collider [2] is designed to detect muons and measure its properties with excellent precision. The muon system consists of 3 complementary gaseous muon detection technologies for triggering and tracking purposes. Drift Tubes are instrumented in the barrel region $(|\eta|<1.2)$, Cathode Strip Chambers in the endcap region $(|\eta|>1.2)$, and Resistive Plate Chambers in barrel and endcap up to $|\eta|<1.6$. The high eta region, $1.6<|\eta|<2.4$ is only instrumented with CSCs. The ongoing Phase-I muon upgrade during the 1st Long Shutdown (LS1) includes the completion of the 4th endcap station with CSCs and RPCs up to $|\eta|<1.8$. The expected upgrade of the LHC during LS3 $(\sim 2022)$ to achieve $\sqrt{s}=7 \mathrm{TeV}$ and $L=5 \times 10^{-34} \mathrm{~cm}^{-2} \mathrm{~s}^{-1}$ will yield a large increase in the particle rate, especially for the endcaps. A L1 trigger system for the silicon tracker will not be installed until LS3. CMS will need additional handles to cope with the high rate of particles between LS2 $(\sim 2018)$ and LS3. The high- $\eta$ region offers a nice opportunity to install additional muon detectors in the currently vacant positions. Naturally, such detectors have to satisfy a high rate capability, $\Omega\left(1 \mathrm{MHz} / \mathrm{cm}^{2}\right)$, a good time resolution for triggering, and a good spatial resolution, $\Omega(100 \mu \mathrm{m})$, for tracking. The current CMS RPC design is not able to sustain the high rates. However, there are various solutions in the field of micro-pattern gaseous detectors (MPGDs), namely gaseous electron multipliers (GEMs) [3]. 


\section{The CMS GEM project}

The baseline of the CMS GEM project is the installation of 36 double-layered triple-GEM chambers in front of the ME1/1 station during LS2, called the GE1/1 system. The chambers will provide full coverage in $\phi$ and $1.55<|\eta|<2.18$ in pseudo-rapidity. The even-numbered GE1/1 chambers will be slightly larger to maximize coverage in $|\eta|$. The station 2 upgrade (GE2/1) consists of installing two rings of double-layered triple-GEM chambers during LS3 covering up to $1.6<|\eta|<2.45$. Each GE2/1 chamber spans about 20 degrees in $\phi$. The third part of the project is a muon near-tagger (ME0) with 18 six-layered triple-GEMs, with each chamber providing coverage of 20 degrees in $\phi$ and $2.0<|\eta|<3.5$ in pseudo-rapidity. The geometry of both GE2/1 and ME0 is yet to be finalized. The location of the proposed GE1/1 and GE2/1 in the present CMS Muon system is shown in figure 1.

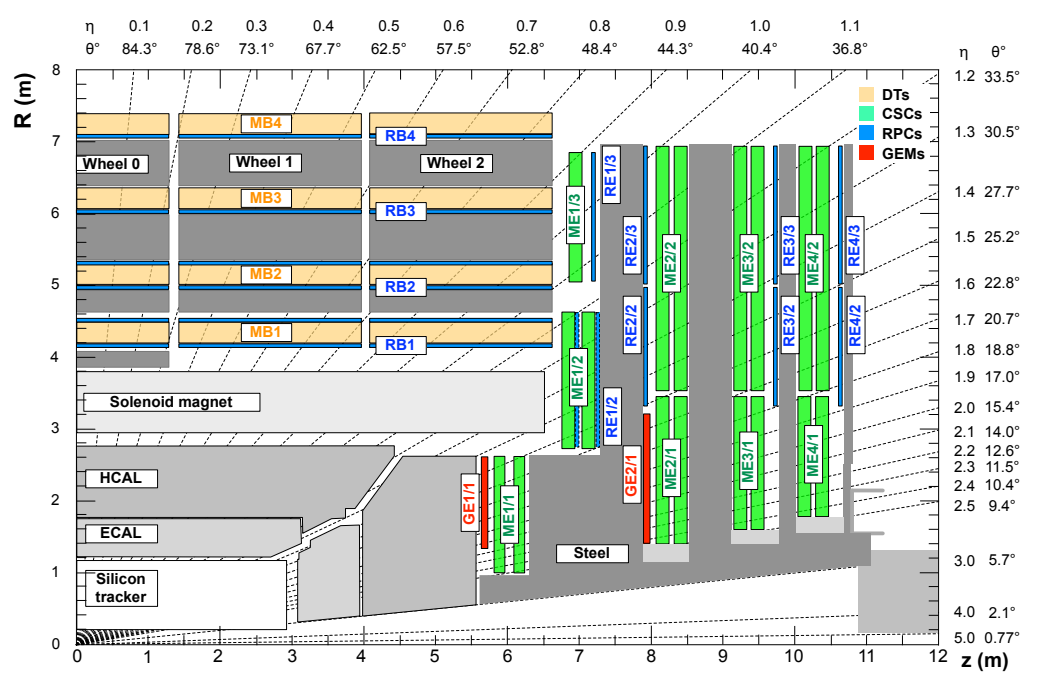

Figure 1. Transverse section of CMS showing the present Muon system with DTs, CSCs and RPCs. The proposed GEM stations, GE1/1 and GE2/1, are given by the red boxes. ME0 is not shown.

\section{Gaseous electron multipliers}

GEMs are a type of MPGDs constructed from a resistive polyimide foil with a thickness of typically $50 \mu \mathrm{m}$, layered between 2 conductive layers (usually copper) with a thickness of about $5 \mu \mathrm{m}$. The foil is perforated with a hexagonal pattern of (bi)conical holes, with a typical hole diameter of $70 \mu \mathrm{m}$ and a pitch of $140 \mu \mathrm{m}$. The foils themselves are produced using a Printed Circuit Board (PCB) manufacturing technique. Many years of R\&D at CERN have made the production of large scale areas $\left(1 \times 2 \mathrm{~m}^{2}\right)$ cost-effective, enabling the design and testing of CMS-scale prototypes. GEMs have an excellent spatial and time resolution, $\Omega(100 \mu \mathrm{m})$ and $\sim 5 \mathrm{~ns}$ respectively. They have a high efficiency (up to $98 \%$ ) and a high rate capability $\Omega\left(1 \mathrm{MHz} / \mathrm{cm}^{2}\right)$. In addition, they are radiation hardened and can be operated with a non-flammable gas mixture of $\mathrm{Ar} / \mathrm{CO}_{2} / \mathrm{CF}_{4}$ (45/15/40), which makes GEMs ideal material for a triggering-tracking detector. At CERN, GEMs have been succesfully used at other experiments (LHCb, COMPASS and TOTEM) in which there was no significant loss of performance, even after many years in operation [4]. 


\section{Impact on trigger and physics impact at the high-luminosity LHC}

\subsection{Trigger}

The expected increase of the LHC beam instantaneous luminosity from the design luminosity of $10^{34} \mathrm{~cm}^{-2} \mathrm{~s}^{-1}$ to maximally $5 \times 10^{34} \mathrm{~cm}^{-2} \mathrm{~s}^{-1}$ in the Phase-II era will have a big impact on the Muon endcap system, in particular the high- $\eta$ region. Many more soft particles will scatter off elastically in the muon detectors and in the particular in the iron return yokes. Multiple scattering causes straightening of the tracks. Low- $p_{\mathrm{T}}$ muons are misinterpreted as high- $p_{\mathrm{T}}$ muons by the CSC Track-Finder, resulting in a flattening of the trigger rate curve at high- $p_{\mathrm{T}}$. To reduce the trigger rate while maintaining high efficiency, one needs to install an additional muon detector (GEM) that measures the bending angle in station 1. The extra measurement points will also yield a higher efficiency, even at scenarios with up to 140 interactions per bunch crossing (pileup).

The proposed GE1/1 chambers are covered with 384 strips, with each strip having a pitch of $\sim 0.5 \mathrm{mrad}$. For trigger purposes, 4 neighboring strips are collected in a OR-ed collection called a GEM-CSC trigger pad, or simply trigger pad. There is nearly $100 \%$ efficiency to reconstruct a single trigger pad. The GE1/1-ME1/1 bending angle provides a clear separation between soft $\left(p_{\mathrm{T}} \sim 5 \mathrm{GeV} / \mathrm{c}\right)$ and hard muons $\left(p_{\mathrm{T}}>20 \mathrm{GeV} / \mathrm{c}\right.$ ) as can be seen in figure 2. A simple GEM-CSC local trigger based on soft stub rejection shows that a rate reduction of a factor 10 can be achieved at an average instantaneous luminosity of $4 \times 10^{34} \mathrm{~cm}^{-2} \mathrm{~s}^{-1}$, equivalent to a pileup of 100 (PU100), see figure 3.

The CSC local trigger has a number of known issues, e.g. stubs are not reconstructed due to an insufficient number of hits, stub timing is incorrect, fake stubs are sent upstream etc. A more advanced integrated GEM-CSC trigger algorithm is being developed to recover stubs in various cases. Preliminary results show an increase of the stub reconstruction efficiency from $90 \%$ to $96 \%$ at PU140. In addition to the GEM-CSC integrated trigger, GEM trigger pads are also to be sent directly to the CSC Track-Finder. Studies to use the trigger pads in the track-fit are currently ongoing. Finally, to make maximal use of the GEM information in the track-fit, a generalized dataformat for the ME1/1 CSC stubs was developed in cooperation with electronics experts.

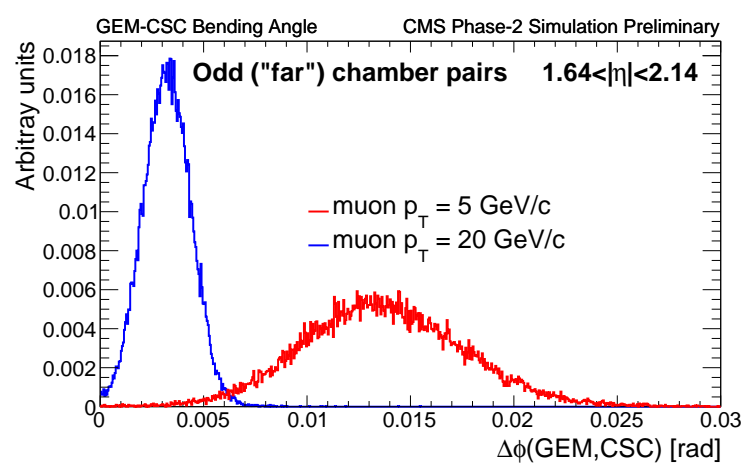

Figure 2. GEM-CSC bending angles for soft $(5 \mathrm{GeV} / \mathrm{c})$ and hard $(20 \mathrm{GeV} / \mathrm{c})$ muons for odd numbered GEM chambers. 

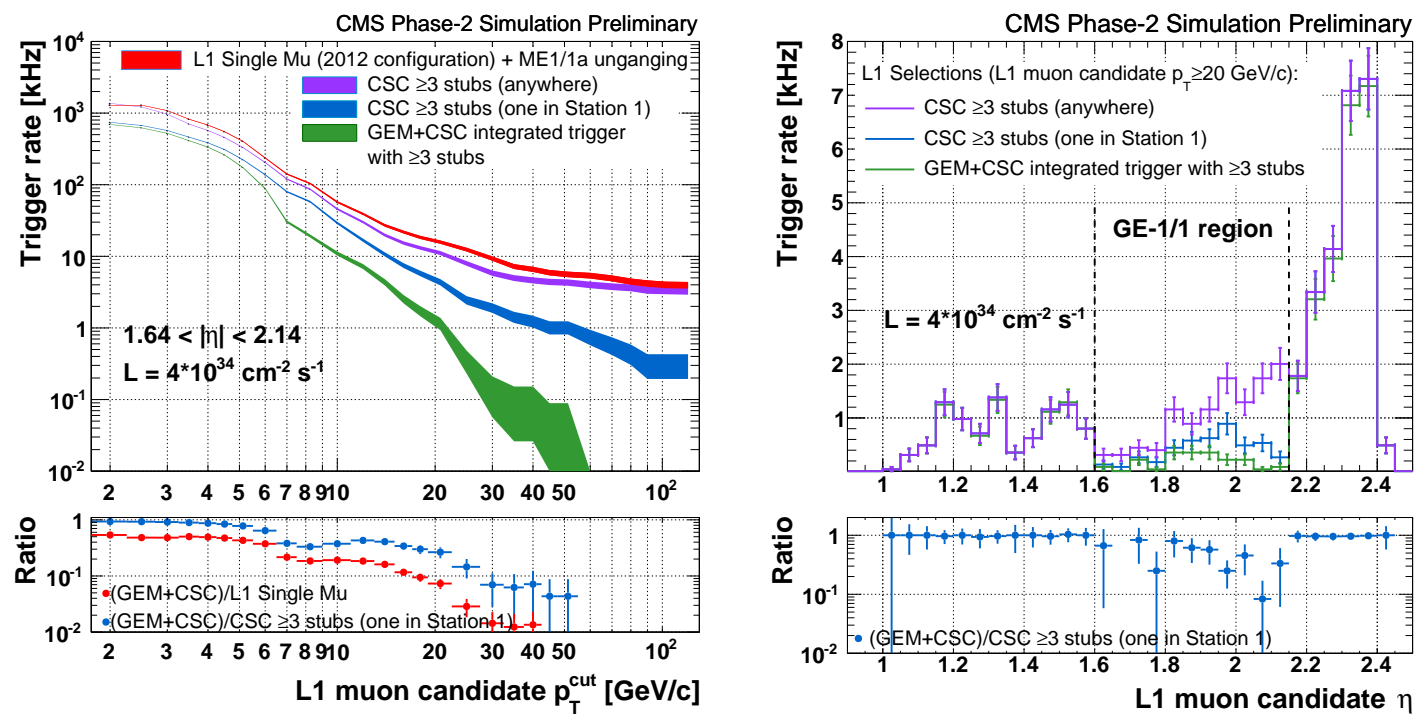

Figure 3. Left: comparison of the trigger rates vs $p_{\mathrm{T}}^{\text {cut }}$ for the Global Muon Trigger in the 2012 configuration and various CSC Track-Finder configurations. Right: comparison of the rates vs $\eta$ for various CSC TrackFinder configurations.

\subsection{Physics}

For physics purposes, the GEMs can provide extra leverage on precision studies of standard model physics, as well as open up a window to explore exotic signatures with muons in the high- $\eta$ region. On standard model physics, GEMs can provide a more precise measurement of the HVV couplings through $H \rightarrow Z Z \rightarrow 4 \mu$ and $\mathrm{H}$ to WW decays, and of the Hff couplings through $H \rightarrow 2 \mu$ and $W H \rightarrow v \mu \tau \tau$. Interesting exotic signatures include $\mathrm{B}_{s}^{0} \rightarrow \mu \mu, Z^{\prime} \rightarrow \mu \mu, W^{\prime} \rightarrow \mu v$ and $\mu^{*} \rightarrow \mu \gamma$.

\section{$5 \quad$ R\&D for CMS GEM project}

\subsection{Early GEM prototypes}

R\&D on GEM detectors for CMS started with the RD51 collaboration in 2009. Various small $10 \times 10 \mathrm{~cm}^{2}$ prototypes $\left(10 \times 10 \mathrm{~cm}^{2}\right)$ with varying geometries, different etching techniques and readout configurations were considered and tested [5].

The major project achievements include the measurement of the single GEM efficiency of $\sim 98 \%$, the time resolution of $4 \mathrm{~ns}$, a spatial resolution of $290 \mu \mathrm{m}$ with a VFAT2 (digital) and $<110 \mu \mathrm{m}$ APV (analog) readout. GEMs have been succesfully operated in a magnetic field, at the CERN SPS, and at FNAL. A gas mixture of $\mathrm{Ar} / \mathrm{CO}_{2} / \mathrm{CF}_{4}$ (45/15/40). In addition, the single mask technology has been proven to be equally effective as the double-mask GEMs.

\subsection{Large GEM prototypes}

Several large GEM prototypes $\left(\sim 1 \times 2 \mathrm{~m}^{2}\right)$ have been developed since 2009 [6, 7]. The first large GEM prototype, GE1/1-I, had all components glued together, had spacers and 8 readout sectors. The number of readout sectors was increased in GE1/1-II to 24. GE1/1-III was the first type to 
make use of the manual stretching technique, see section 5.3. In addition, the distances of the drift, transfer and induction region were finalized. GE1/1-IV was the first prototype to be fully mechanically constructed and produced at various production sites around the world. The final version GE1/1-V will have an optimized coverage in $\eta$ and $\phi$ and will have short and long types. Figure 4 shows the 2010 and 2013 large GEM detector prototypes.
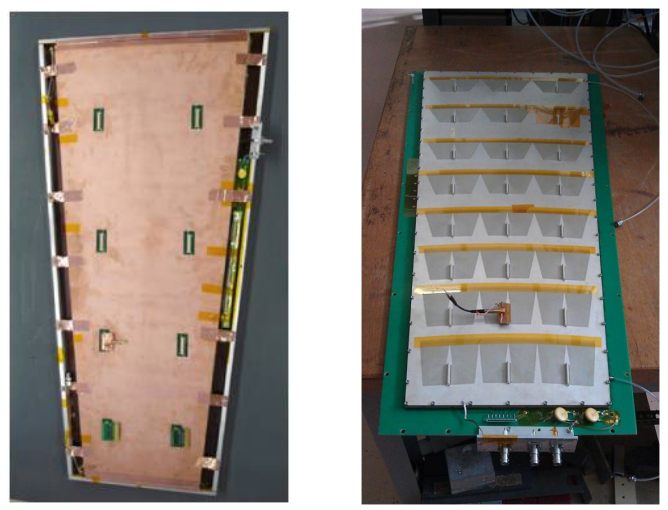

Figure 4. Left: first large GEM type detector produced in 2010. Right: GE1/1-IV produced in 2013.

\subsection{GEM foil stretching}

A flat GEM foil is essential for a uniform chamber response. Initially, the GEM foils were stretched by heating the foils in a special oven and gluing them onto a spacer frame. This procedure was cumbersome and introduced less sensitive areas in the fiducial volume. A second method involved the in-situ thermal stretching developed at Florida Institute of Technology (FIT) using $16125 \mathrm{~W}$ heat lamps. Eventually the simplest and most cost-effective method proved to be manual stretching. A purely mechanical construction of o-rings and screws on a plastic (FR4) frame attached to the GEM foil allows to adjust the tension accordingly. Moreover, the construction permits a fast and easy repair of broken chambers. A close-up of the frame is shown in figure 5.

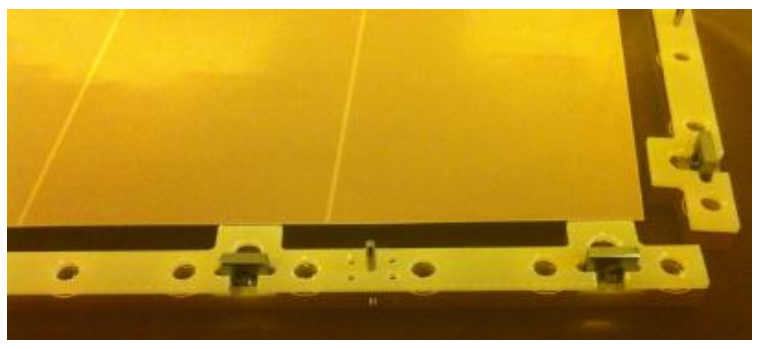

Figure 5. Close-up of the plastic frame for the manual stretching.

\subsection{Aging tests}

The GEM detectors are designed to sustain the harsh environment for long term operation $\Omega$ (10 years). Long term operation of GEMs at LHCb, COMPASS, TOTEM has shown no sign of peformance degradation. Nevertheless, it is important to monitor the GE1/1-V performance 
with integrated charge collection to ensure long term operation, understand material degradation and propose solutions to counter it. To this end, gamma irradiation tests using 662 photons from ${ }^{137} \mathrm{Cs}$ source (566 GBq) are ongoing at the CERN GIF and are planned at the GIF++ lab [8].

\subsection{Chamber construction and quality control}

The GEM chamber construction procedure has been optimized over the past couple of years. The assembly itself contains 3 major steps. The first step is the preparation of the drift board. The PCB is equiped with metallic inserts and HV probes, and is fixed to an outer frame. The second step is the prepartion of the GEM stack. Three GEM foils are sandwiched in between platic frames. In the final step the GEM stack is placed on top of the drift board. The electrical contacts and HV divider are installed, after which the chamber is closed with the readout PCB. Finally, the gas in- and outlets are inserted. With the help of the manual stretching and the purely mechanical construction the procedure has optimized to take less than 2 hours instead of $\sim 1$ week with the gluing principle. The chamber construction is followed by a standardized quality procedure. During 20136 GE-IV prototypes have been built at various sites: INFN Frascati, INFN Bari, FIT, CERN, UGent and BARC.

\subsection{Data acquisition and electronics}

The GEM readout electronics is divided into the on-chamber and off-chamber electronics, as can be seen in figure 6. On-chamber, the GE1/1 detector prototype are first subdivided in 8 eta partitions. Each eta partition is further subdivided in 3 readout sectors along $\phi$. Each sector is covered with 128 readout strips which form the inputs to the 30 front-end ASIC (VFAT3 chip) via a connector on the GEM readout board (GEB). An Optohybrid board plugs into the GEB which contains the Gigabit Transceiver chip set, a Field programmable Gate Array, and optical receivers and transmitters to provide 2 optical paths to the off-detector electronics. The first path sends the fixed latency trigger data from the GEMs to the CSCs. The second path is bidirectional and runs between the $\mu$ TCA (micro Trigger Control and Acquisition) crates in the counting room and the optohybrid. It is used for sending set-up and control signals to the front-end chips.

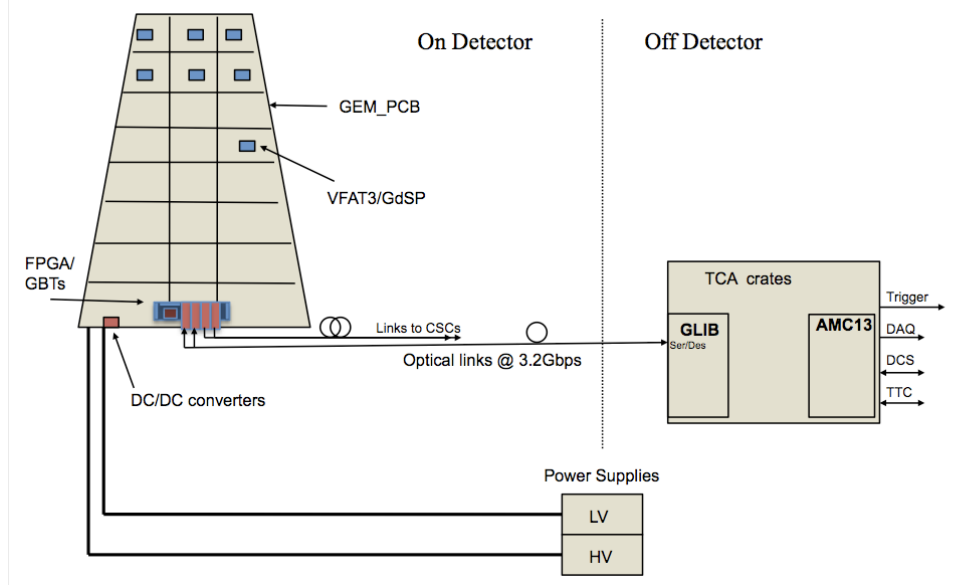

Figure 6. Schematic overview of the on- and off-detector electronics for the GEM chambers. 


\section{Integration into CMS}

With the prospective of the full installation of GE1/1 during LS2, a slice test will take place during the Year-End Technical Stop of 2016. A small number of fully working GE1/1 chambers will be installed in one of the endcaps with a total coverage from 10 to 20 degrees. In preparation of the slice test, 3 dummy chambers (exact dimensions and weight, but no electronics) were produced during May 2013. The subsequent trial installation of the dummy chambers during the Summer of 2013 (figure 7) allowed the technicians to work out the cable routing, layout of the cooling pipes, hardware infrastructure etc. A new series of dummies, based on updated chamber dimensions, were produced during winter 2014 , see figure 8 . A new trial installation will take place before the end of LS1.
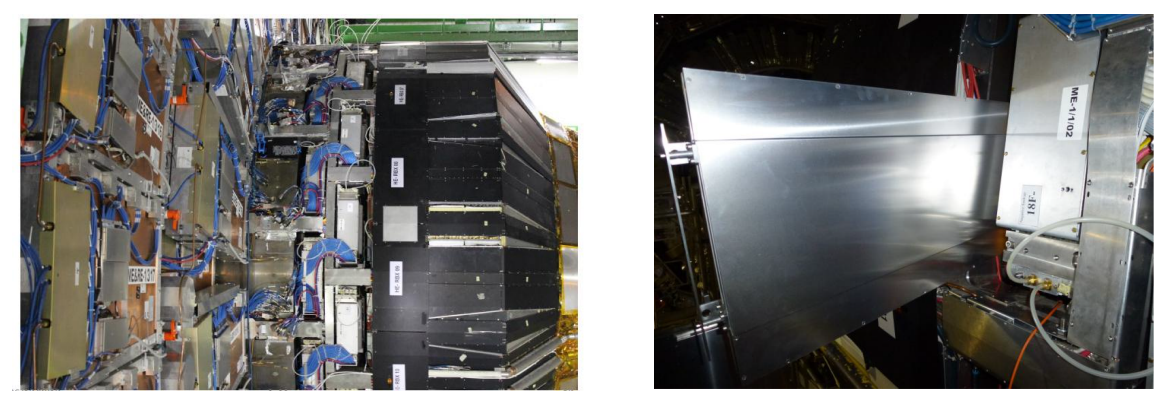

Figure 7. Left: location of the GE1/1 slots in the Muon endcap nose. Right: insertion of the dummy chambers.

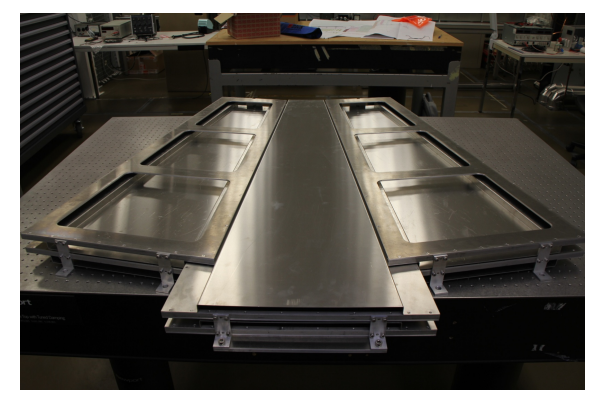

Figure 8. New version of dummy chambers according to long and short baseline.

\section{Conclusions and outlook}

The R\&D for the CMS GEM project started almost 5 years ago (2009) with the RD51 Collaboration at CERN. Since then, triple-GEM technology has matured enough to satisfy the physics and trigger needs for CMS in the post-LS2 era. The present GE1/1-V type is fully compliant with the restrictions for installation in the cavern. A partial approval for the installation of the GE1/1 system was obtianed during Summer 2013. Currently, CMS GEM collaboration is preparing a Technical Design Report for GE1/1 by October 2014. The production of the finalized GE1/1-V detectors is scheduled for 2014-2015. During the Year-End Technical Stop of 2016-2017, a slice test will take place involving a small number of GEM super chambers installed in YE1/1. 


\section{Acknowledgments}

We acknowledge the support from the RD51 collaboration and CERN.

\section{References}

[1] CMS collaboration, The CMS experiment at the CERN LHC, 2008 JINST 3 S08004.

[2] L. Evans and P. Bryant, LHC Machine, 2008 JINST 3 S08001.

[3] F. Sauli, GEM: A new concept for electron amplification in gas detectors, Nucl. Instrum. Meth. A 386 (1997) 531.

[4] A. Cardiniet et al., The Operational Experience of the Triple-GEM Detectors of the LHCb Muon System: Summary of 2 Years of Data Taking, IEEE Nucl. Sci. Symp. Conf. Rec. 2012 (2012) 759;

B. Ketzer, Status of the PixelGEM Detectors, COMPASS Technical Board Meeting (2011), unpublished;

B. Ketzer et al., A fast tracker for COMPASS based on the GEM, Nucl. Phys. Proc. Suppl. 125 (2003) 368.

[5] D. Abbaneo et al., Characterization of GEM Detectors for Application in the CMS Muon Detection System, IEEE Nucl. Sci. Symp. Conf. Rec. 2010 (2010) 1416 [arXiv: 1012.3675].

[6] D. Abbaneo et al., Construction of the rst full-size GEM-based prototype for the CMS high- $\eta$ muon system, IEEE Nucl. Sci. Symp. Conf. Rec. 2010 (2010) 1909, RD51 Note 2010-008 [arXiv: 1012.1524].

[7] M. Tytgat et al., Construction and Performance of Large-Area triple-GEM Prototypes for Future Upgrades of the CMS Forward Muon System, IEEE Nucl. Sci. Symp. Conf. Rec. 2011 (2011) 1019, RD51 Note 2011-012 [arXiv: 1111.7249].

[8] D. Abbaneo et al., The status of the GEM project for CMS high- $\eta$ muon system, Nucl. Instrum. Meth. A 732 (2013) 203.

[9] P. Vichoudis et al., The Gigabit Link Interface Board (GLIB), a exible system for the evaluation and use of GBT-based optical links, 2010 JINST 5 C110167. 